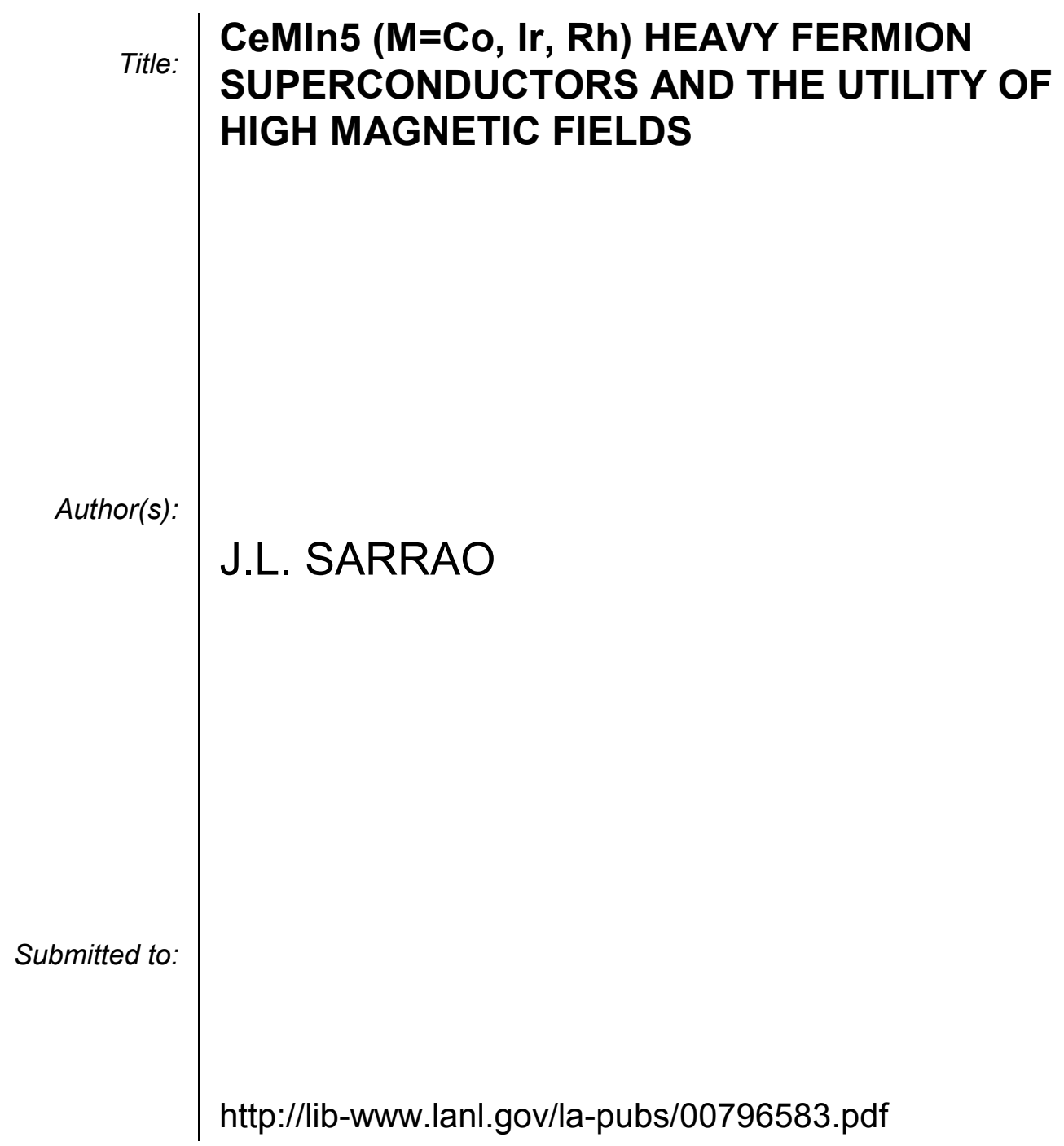

Los Alamos National Laboratory, an affirmative action/equal opportunity employer, is operated by the University of California for the U.S. Department of Energy under contract W-7405-ENG-36. By acceptance of this article, the publisher recognizes that the U.S. Government retains a nonexclusive, royaltyfree license to publish or reproduce the published form of this contribution, or to allow others to do so, for U.S. Government purposes. Los Alamos National Laboratory requests that the publisher identify this article as work performed under the auspices of the U.S. Department of Energy. Los Alamos National Laboratory strongly supports academic freedom and a researcher's right to publish; as an institution, however, the Laboratory does not endorse the viewpoint of a publication or guarantee its technical correctness. 


\title{
CeMIn $_{5}(\mathrm{M}=\mathrm{Co}$, Ir, Rh) HEAVY FERMION SUPERCONDUCTORS AND THE UTILITY OF HIGH MAGNETIC FIELDS
}

\author{
J.L. SARRAO \\ Los Alamos National Laboratory, Los Alamos, NM 87545, USA \\ E-mail: sarrao@lanl.gov
}

\begin{abstract}
We review the properties of the recently discovered $\mathrm{CeMIn}_{5}(\mathrm{M}=\mathrm{Co}, \mathrm{Ir}, \mathrm{Rh})$ heavy fermion superconductors and discuss the present state of our understanding of these materials. A particular focus is the role that magnetic fields have played in elucidating the properties of these materials. Specifically, we discuss quantum oscillation measurements on CeMIn 5 , the influence of applied field on the linear coefficient of specific heat, $\gamma$, and the nature of the H$\mathrm{T}$ phase diagrams in both the normal and superconducting states of these materials.
\end{abstract}

\section{Introduction}

The response of heavy fermions to applied magnetic fields has significantly contributed to our understanding of these materials [1]. The recent availability of magnetic fields on the order of 100 Tesla, which corresponds to Zeeman energies on the order of $100 \mathrm{~K}$, a scale which often exceeds those characteristic of heavy fermion ground states and a growing suite of available measurement techniques only serves to increase this impact [2]. In particular, the dependence of the conduction electron effective mass, most simply probed by measurements of the linear-in-T coefficient of the low-temperature heat capacity, on applied field allows one to separate mass enhancements due to spin fluctuation behavior from 'real' heavy fermion character [3]. Furthermore, the appearance, or not, of field-induced metamagnetic transitions in heavy fermion compounds allows insights to be gained into the degree of f-electron localization or itineracy, an open and material-specific issue in heavy fermions [4]. Finally, at least in some circumstances, the availability of high magnetic fields facilitates quantum oscillation studies of the fermi surfaces of these materials [5]. In what follows, we review the recent discovery of the $\mathrm{CeMIn}_{5}$ family of heavy fermion compounds and discuss the ways in which fielddependent measurements have contributed to our understanding of these materials.

\section{Basic properties of $\mathrm{CeMIn}_{5}$}

The $\mathrm{CeMIn}_{5}(\mathrm{M}=\mathrm{Co}$, Ir, Rh) family of compounds are the first heavy fermion materials in which structural layering can be demonstrated to play an important role. Each of these materials is a tetragonal derivative of $\mathrm{CeIn}_{3}$, and their ground state properties depend on both the effective hydrostatic and uniaxial pressure created by 
the tetragonal stacking of $\mathrm{CeIn}_{3}$ and $\mathrm{MIn}_{2}$ layers. Co, Ir, and $\mathrm{Rh}$ are isovalent so, although these materials bear remarkable similarities to the cuprates, carrier-density tuning does not play an explicit role in determining their ground state properties.

At ambient pressure, CeRhIn ${ }_{5}$ orders antiferromagnetically at $3.8 \mathrm{~K} . \mathrm{T}_{\mathrm{N}}$ increases weakly with applied pressure, an unconventional dependence, until it vanishes abruptly near $16 \mathrm{kbar}$, at which point superconductivity, with a $\mathrm{T}_{\mathrm{c}}$ of $2.1 \mathrm{~K}$, is observed [6]. The superconductivity is bulk as evidenced by heat capacity [7] and nuclear magnetic resonance [8,9], and the observed temperature dependencies are consistent with unconventional, $d$-wave pairing. The value of $C / T$ just above $T_{N}$ and $\mathrm{T}_{\mathrm{c}}$ is $\sim 400 \mathrm{~mJ} / \mathrm{molK}^{2}$ in both cases, confirming that CeRhIn ${ }_{5}$ is a heavy fermion.

CeIrIn ${ }_{5}$ displays bulk superconductivity at $0.4 \mathrm{~K}$ that develops out of a state with $\gamma \sim 700 \mathrm{~mJ} / \mathrm{molK}^{2}$ [10]. Again, heat capacity, thermal transport, and nuclear magnetic resonance measurements provide strong evidence for line nodes in the superconducting gap [11-13]. An interesting wrinkle regarding $\mathrm{CeIrIn}_{5}$ is that its resistivity vanishes near $1 \mathrm{~K}$, well above the bulk $\mathrm{T}_{\mathrm{c}}$. The detailed origin of this filamentary state remains unclear [14], but it is robust as a function of applied magnetic field and displays the same crystallographic anisotropy as the bulk transition. Furthermore, the zero-resistance transition in $\mathrm{CeIrIn}_{5}$ 'reveals' the value of bulk $\mathrm{T}_{\mathrm{c}}$ that can be approached with either pressure [15] or chemical doping [16].

With an ambient-pressure $\mathrm{T}_{\mathrm{c}}$ of $2.3 \mathrm{~K}, \mathrm{CeCoIn}_{5}$ displays the highest transition temperature of any heavy Fermion superconductor [17]. Again, the implication is dwave superconductivity $[11,13,18]$. Applied pressure can further increase $T_{c}$ to 2.6 $\mathrm{K}$ (at a pressure of $15 \mathrm{kbar}$ ) [19]. The observed $\mathrm{C} / \mathrm{T}$ of $300 \mathrm{~mJ} / \mathrm{molK}^{2}$ at $\mathrm{T}_{\mathrm{c}}$ is rather modest, but the application of a magnetic field greater than $\mathrm{Hc}_{2}$ reveals that $\gamma$ is not fully developed at $\mathrm{T}_{\mathrm{c}}(\mathrm{H}=0)$, and, in fact, the field-induced normal state $\gamma \sim 1000$ $\mathrm{mJ} / \mathrm{molK}^{2}$ is quite large.

An additional surprising feature of $\mathrm{CeMIn}_{5}$ is the robustness of intermediate alloys, e.g., $\mathrm{CeM}_{1-\mathrm{x}} \mathrm{M}_{\mathrm{x}} \mathrm{In}_{5}\left(\mathrm{M}\right.$ or $\left.\mathrm{M}^{\prime}=\mathrm{Co}, \mathrm{Ir}, \mathrm{Rh}\right)$ [20]. Single crystals can be grown for the full range of $\mathrm{x}$ for all $\mathrm{M}$ and $\mathrm{M}$ ' without apparent degradation of sample quality; neither clustering nor gross phase segregation has been observed. Thus, one is presented with the opportunity of exploring the competition and interaction of diverse ground states, e.g., magnetism and superconductivity $[16,20,21]$. The range of $\mathrm{x}$ for which superconductivity can be observed is remarkable, $\sim 0.7$ for Rh-Ir alloys, and is rivaled only by the cuprates in terms of the flexibility of chemical tuning.

sarrao-pphmf.doc submitted to World Scientific 1/29/02 : 2:25 PM 


\section{Trends in electronic anisotropy deduced from dHvA measurements}

\subsection{Fermi surface evolution with $M$}

Because of the high quality of single crystals that result from flux growth-based crystal growth, substantial progress has been made in quantum oscillation studies of $\mathrm{CeMIn}_{5}$ [22-26]. From a broad perspective, reasonable agreement is found between LDA band structure calculations and de Haas-van Alphen oscillation measurements. Three pieces of fermi surface (FS) are calculated and observed, a relatively small quasi-spherical sheet, a quasi-cylindrical sheet, and a third more complex, largervolume sheet. More important are the trends that emerge on evolution from $\mathrm{CeRhIn}_{5}$ to $\mathrm{CeIrIn}_{5}$ to CeCoIn ${ }_{5}$. As the ambient pressure ground state evolves from antiferromagnet to low-T superconductor to higher-T superconductor, the degree of quasi-2d character - thought to play an important role in the enhanced $T_{c}$ of these materials relative to $\mathrm{CeIn}_{3}$ - increases substantially, as judged by the decreased variation in extremal areas measured on the quasi-cylindrical FS sheet. Furthermore, increased effective masses, consistent with the known increases in $\mathrm{C} / \mathrm{T}$, are observed. However, the heaviest orbits and M-dependent mass enhancement are not observed on the quasi-cylindrical FS, but rather on the larger piece of FS, complicating the interpretation that high- $T_{c}$ heavy fermion superconductivity derives from the quasi-2d character of these materials.

\subsection{Degree of f-electron localzation}

It has also proved possible to observe quantum oscillations in a wide range of alloys, in particular $\mathrm{Ce}_{1-\mathrm{x}} \mathrm{La}_{\mathrm{x}} \mathrm{MIn}_{5}$ [27,28]. Such measurements provide a model-free measure of the degree of f-electron localization in heavy fermion materials. Because Ce possesses one f-electron whereas La has none, replacing Ce with La should result in a change in FS volume if the f-electron is itinerant and contributes to the FS. On the other hand, if the f-electron is highly localized, no such change in FS volume is expected. Similar La-dilution studies have been performed in a wide range of heavy fermion materials, and a general trend in which heavy fermion antiferromagnets possess localized f-electrons and heavy fermion superconductors possess itinerant $\mathrm{f}$ electrons seems to emerge. Our measurements of $\mathrm{Ce}_{1-x} \mathrm{La}_{x} \mathrm{RhIn}_{5}$ may confirm this trend [27]. Both the observed dHvA frequencies, which measure the FS area, and the measured effective masses depend only weakly on La concentration, suggesting highly localized f-electrons. However, although the data are not as complete, $\mathrm{CeIrIn}_{5}$ and $\mathrm{CeCoIn}_{5}$ appear to display a similar degree of localization [28]. This strong degree of f-electron localization is also revealed by the bulk magnetic properties of $\mathrm{CeMIn}_{5}$, which appear to be dominated by crystal-electric-field effects $[29,30]$. Interestingly, preliminary dHvA measurements as a function of pressure in $\mathrm{CeRhIn}_{5}$, in which the observed effective masses and, perhaps, the extremal areas are seen to vary near the antiferromagnetic-superconducting transition, reveal a

sarrao-pphmf.doc submitted to World Scientific 1/29/02 : 2:25 PM 
greater degree of variation in f-electron localization [31]. Unfortunately, although data exist [32], insufficient progress has been made in $\mathrm{CeM}_{1-\mathrm{x}} \mathrm{M}_{\mathrm{x}}{ } \mathrm{In}_{5} \mathrm{dHvA}$ studies to determine whether similar changes are observed as a function of doping.

\section{H-T phase diagram in $\mathrm{CeCoIn}_{5}$}

Partially in the process of performing dHvA measurements in $\mathrm{CeCoIn}_{5}$, a remarkable H-T phase diagram has emerged [33]. CeCoIn ${ }_{5}$ possesses upper critical fields that are very large and anisotropic. For a $\mathrm{T}_{\mathrm{c}}$ of $2.3 \mathrm{~K}$, simple estimates predict a critical field of $\sim 4.6 \mathrm{~T}$ [34]. $\mathrm{CeCoIn}_{5}$, however, displays $\mathrm{Hc}_{2}[001] \sim 5 \mathrm{~T}$ and $\mathrm{Hc}_{2}[100] \sim 12 \mathrm{~T}$. Furthermore, the angular and temperature evolution of these critical fields is anomalous [33,35]. When field is applied along the c-axis, firstorder transitions are observed in temperature and field sweeps for $4.6 \mathrm{~T}<\mathrm{H}<\mathrm{Hc}_{2}$ in thermal expansion and specific heat data, suggesting Pauli limiting [36].

The in-plane behavior of $\mathrm{Hc}_{2}$ in $\mathrm{CeCoIn}_{5}$ is even more anomalous [33]. Rotating the applied field from [001] into the plane at lowest temperatures reveals different behaviors for rotation into [100] and [110], respectively. The angular variation of $\mathrm{Hc}_{2}$ from [001] to [100] is that expected from the anisotropic effective masses deduced from quantum oscillation measurements. [001]-[110] rotations, on the other hand, follow a form expected for thin films rather than 3-d superconductors and suggest 2-d character. The temperature evolution of $\mathrm{Hc}_{2}$ for field applied along [110] is even more anomalous. For $\mathrm{T}<0.6 \mathrm{~T}_{\mathrm{c}}$, a step-like transition in magnetization is observed at $\mathrm{Hc}_{2}$ (determined resistively) that, for decreasing field sweeps, remains step-like but occurs at fields lower than $\mathrm{Hc}_{2}$. For $\mathrm{T}>0.6 \mathrm{~T}_{\mathrm{c}}$, the conventional behavior is recovered, the magnetization vanishes smoothly at $\mathrm{Hc}_{2}$ independent of field-sweep direction. These observations provide strong evidence for the realization of an inhomogeneous Fulde-Ferrell-Larkin-Ovchinikov (FFLO) state in $\mathrm{CeCoIn}_{5}$.

A metamagnetic transition is also observed in the normal state of CeCoIn 5 . For temperatures at least as high as $25 \mathrm{~K}$, and extending down to $0.6 \mathrm{~T}_{\mathrm{c}}$ in the field induced normal state, a step-like transition is observed in magnetization for fields near $9 \mathrm{~T}$. The positive step in magnetization suggests the possibility of fieldinduced ferromagnetism. Although the detailed nature of this transition remains unclear, a (partial) spin polarization of the FS is a possibility. This metamagnetic transition also might be related to similar phenomena observed in CeIrIn ${ }_{5}$, albeit at higher fields, by Stewart et al. [37]. Finally, it is worth noting that the FFLO behavior observed in the superconducting state emerges from the field-induced ferromagnetic state and may, in fact, be stabilized by it.

sarrao-pphmf.doc submitted to World Scientific 1/29/02 : 2:25 PM 


\section{General trends in applied fields}

Finally, we discuss the field-dependent evolution of the heavy fermion state itself in $\mathrm{CeMIn}_{5}$. Measurements of heat capacity in fields as high as $28.5 \mathrm{~T}$ reveal that $\gamma$ is suppressed by $33 \%$ and $50 \%$ in CeIrIn 5 and $\mathrm{CeCoIn}_{5}$, respectively [38]. Although the suppression of $\gamma$ is significant, substantial mass enhancement does persist to high fields, consistent with observations from dHvA measurements, confirming that the enhanced heat capacity is attributable to heavy fermion behavior rather than magnetic correlations resulting from proximity to a phase transition. Because $T_{N}$ depends only weakly on field in CeRhIn ${ }_{5}$, similar estimates of $\gamma(\mathrm{H})$ are difficult, but it is clear that heavy-fermion mass enhancement comparable to that of CeIrIn ${ }_{5}$ and $\mathrm{CeCoIn}_{5}$ are present in $\mathrm{CeRhIn}_{5}$. Another normal-state feature of $\mathrm{CeMIn}_{5}$ revealed by high-field measurements is the ubiquitous presence of metamagnetic transitions. $\mathrm{CeRhIn}_{5}$ [22,39], CeIrIn ${ }_{5}$ [37] and $\mathrm{CeCoIn}_{5}$ [33] all display field-induced transitions in heat capacity or magnetization.

\section{Conclusions}

In summary, high magnetic field measurements have played an important role in our present understanding of the $\mathrm{CeMIn}_{5}$ family of heavy fermion superconductors. Heat capacity measurements as a function of field reveal that the low- $\mathrm{T}$ mass enhancement is due to heavy fermion behavior. Quantum oscillation measurements confirm this point and further reveal trends as a function of $\mathrm{M}$ that suggest that a quasi two-dimensional electronic structure exists and may play a role in determining $\mathrm{T}_{\mathrm{c}}$ in these materials. Finally, applied fields provide another thermodynamic axis along which ground-state properties can be mapped. Not only are metamagnetic transitions observed in the paramagnetic states of $\mathrm{CeIrIn}_{5}$ and $\mathrm{CeCoIn}_{5}$ but also anomalous evolution of the Neel state in $\mathrm{CeRhIn}_{5}$ as well as candidate FFLO behavior in the superconducting state of $\mathrm{CeCoIn}_{5}$ is revealed.

\section{Acknowledgements}

I thank R. G. Goodrich, D. Hall, N. Harrison, T. P. Murphy, E. C. Palm, G. R. Stewart, J. D. Thompson, and S. W. Tozer for important contributions to this effort. Work at Los Alamos was performed under the auspices of the U.S. Department of Energy. Work at the NHMFL, where many of the high-field measurements discussed above were performed, is supported by the N.S.F. and the State of Florida. 


\section{References}

1. Andraka B., et al., Phys. Rev. B 39, 6420 (1989).

2. Jaime M., et al, Nature 405, 160 (2000).

3. Stewart G.R., et al., Phys. Rev. B 37, 3344 (1988).

4. Schroder A., et al., Nature 413, 297 (2000).

5. Goodrich R. G., et al., Phys. Rev. Lett. 82, 3669 (1999).

6. Hegger H., et al., Phys. Rev. Lett. 84, 4986 (2000).

7. Fisher R.A., et al., submitted to Phys. Rev. Lett. (2001); cond-mat/0109221.

8. Mito T., et al., Phys. Rev. B 63, 220507 (2001).

9. Kohori Y., et al., Eur. Phys. J. B 18, 601 (2000).

10. Petrovic C., et al., Europhys. Lett. 53, 354 (2001).

11. Movshovich R., et al., Phys. Rev. Lett. 86, 5152 (2001).

12. Zheng G.Q., et al., Phys. Rev. Lett. 86, 4664 (2001).

13. Kohori Y., et al., Phys. Rev. B 64, 134526 (2001).

14. Bianchi A., et al., to appear in Phys. Rev. B (2001); cond-mat/0108081.

15. Sparn G., et al., to appear in Physica B (2001).

16. Pagliuso P.G., et al., Phys. Rev. B 64, 100503 (2001).

17. Petrovic C., et al., J. Phys.: Condens. Matter 13, L337 (2001).

18. Izawa K., et al., Phys. Rev. Lett. 87, 057002 (2001).

19. Nicklas M., et al., J. Phys.: Condens. Matter 13, L905 (2001).

20. Pagliuso P.G., et al., to appear in Physica B (2001); cond-mat/0107266.

21. Zapf V.S., et al., to appear in Phys. Rev. B (2001).

22. Cornelius A.L., et al., Phys. Rev. B 62, 14181 (2001).

23. Hall D., et al., Phys. Rev. B 64, 064506 (2001).

24. Haga Y., et al., Phys. Rev. B 63, 060503 (2001).

25. Hall D., et al., to appear in Phys. Rev. B (2001); cond-mat/0102533.

26. Settai R., et al., J. Phys.: Condens. Matter 13, L627 (2001).

27. Alver U., et al., Phys. Rev. B 64, 180402 (2001).

28. Goodrich R.G., et al., unpublished (2001).

29. Curro N.J., et al., Phys. Rev. B 64, 180514 (2001).

30. Pagliuso P.G., et al., to appear in Physica B (2001).

31. Onuki Y., private communication (2001).

32. Goodrich R.G., et al., unpublished (2001).

33. Murphy T.P., et al., submitted to Phys. Rev. Lett. (2001); cond-mat/0104179.

34. Clogston A. M., Phys. Rev. Lett. 9, 266 (1962).

35. Oeschler N., et al., unpublished (2001).

36. Bianchi A., et al., unpublished (2001).

37. Stewart G.R., et al., these proceedings.

38. Kim J.S., et al., Phys. Rev. B 64, 134524 (2001).

39. Takeuchi T., et al., J. Phys. Soc. Jpn. 70, 877 (2001). 